\title{
Propuesta curricular de habilidades adaptativas para la estimulación temprana de niños y niñas de 0 a 3 años de edad, con discapacidad cognitiva
}

\author{
María Guillermina Jara Ocampo ${ }^{l}$ \\ Centro de Enseñanza Especial de Heredia \\ Heredia, Costa Rica \\ Pahola Roda Fuentes ${ }^{2}$ \\ Centro de Enseñanza Especial de Heredia \\ Heredia, Costa Rica
}

Recibido 17 de abril de 2009• Aceptado 02 de diciembre de 2009

\begin{abstract}
Resumen. En el estudio desarrollado se fundamenta una Propuesta curricular basada en las habilidades adaptativas para la estimulación temprana de niños y niñas de 0 a 3 años de edad, con discapacidad cognitiva. La Propuesta tiene la intención de mejorar la coherencia, la pertinencia y la efectividad de la educación especial que se ofrece a estudiantes con necesidades educativas especiales, específicamente en condición de discapacidad cognitiva desde la estimulación temprana con población de 0 a 3 años de edad. El objeto de estudio surge del contexto de trabajo, es decir, de las necesidades individuales del docente de educación especial y de los y las estudiantes.
\end{abstract}

Palabras clave. Currículo, atención integral, habilidad adaptativa, conducta adaptativa, educación especial, familia.

Abstract. The study developed builds a curriculum proposal based on adaptive skills for early stimulation of children from 0 to 3 years with cognitive disabilities as a final requirement for graduation from the Masters in Education with Emphasis on Development The proposal intends to improve the coherence, relevance and effectiveness of special education offered to students with special educational needs, specifically with cognitive impairment conditions, from early stimulation with a population of 0 to 3 years. The main objective of the study rises from the working context of the study: the individual needs of special education teachers and their students.

Key words. Curricullum, integral atention, adaptive skills, adaptive behaviors, special education, family.

\footnotetext{
${ }^{1}$ Bachiller en Ciencias de la Educación Especial con énfasis en Retardo Mental de la Universidad de Costa Rica (UCR), Máster en Pedagogía con énfasis en Desarrollo y Atención Integral de la Primera Infancia de la Universidad Nacional (UNA), Costa Rica. Tiene 24 años de experiencia laboral de niños y niñas con retardo mental, de los cuales los últimos 14 años han sido en estimulación temprana, en el Centro de Enseñanza Especial de Heredia, Costa Rica.Correo electrónico: guillerminajarao@hotmail.com

${ }^{2}$ Bachiller en Ciencias de la Educación Especial de la Universidad de Costa Rica (UCR), Máster en Pedagogía con énfasis en Desarrollo y Atención Integral de la Primera Infancia de la Universidad Nacional (UNA), Costa Rica. Con tres años de experiencia laboral. Actualmente labora en el Centro de Enseñanza Especial de Heredia, Costa Rica, en el nivel de preparatoria, para la población con Retardo Mental. Correo electrónico: pfuentes1412@hotmail.com
}

La Revista Electrónic@ Educare está indizada en el Catálogo LATINDEX. 
El viaje real del descubrimiento no consiste en buscar nuevas tierras, sino verlas con nuevos ojos.

Marcel Proust

\section{Introducción}

El proyecto nace de una inquietud de las autoras, ante la necesidad de ofrecer una mayor coherencia en la programación educativa que se ofrece a los infantes de cero a tres años de edad, que presentan una discapacidad cognitiva y que asisten a un servicio de estimulación temprana en un centro de enseñanza especial de carácter público, y que sea propia y contextualizada a la realidad inmediata en esta área de la especialidad.

Los años de trabajo con esta población han permitido vislumbrar que no existe una misma línea de programación educativa que sea consecuente con los niveles por los que pasa el estudiante con discapacidad cognitiva, esto es: desde la estimulación temprana hasta un IV ciclo, en el que se da una preparación con un énfasis vocacional.

El Ministerio de Educación Pública (MEP) determina, por medio de sus Normas y Procedimientos para el manejo técnico-administrativo de los servicios educativos para estudiantes con retardo mental, redactadas en el año 2005, que el nivel de estimulación temprana lleve a cabo su programación educativa con base en la "Guía Curricular para la estimulación del desarrollo integral del niño menor de seis años", la cual fue elaborada, en 1982, por la Caja Costarricense de Seguro Social y el Patronato Nacional de la Infancia, estructurada en tres áreas, a saber: psicomotora, socio afectiva y cognitiva.

Para los niveles de Educación Preescolar y Educación con énfasis en I y II ciclos, el MEP dispone que se adapten los programas curriculares diseñados para la educación regular, de acuerdo con las necesidades educativas especiales de cada estudiante. Para los niveles de III y IV ciclos, establece que la programación se realice con base en habilidades adaptativas.

El interés de la investigación no sólo es el evidenciar la desarticulación existente entre los abordajes que se llevan a cabo en los procesos de enseñanza y de aprendizaje de esta población, lo cual ocasiona que al llegar a los niveles superiores, no cuentan con las herramientas necesarias para una preparación vocacional funcional, sino que, también, se busca potenciar y dar herramientas pedagógicas al docente para que analice y sistematice su práctica cotidiana, para lo cual debe poder investigar los contextos en que se materializa la aplicación de la propuesta educativa, así como los diversos actores involucrados.

\section{Un punto de partida}

La investigación se llevó a cabo en el Centro de Enseñanza Especial de Heredia, en donde se tomó como sujetos de estudio a los estudiantes del nivel de estimulación temprana.

Los estudiantes pertenecen a todos los cantones de la provincia de Heredia, excepto el cantón de Sarapiquí, esto a causa de su lejanía respecto al centro educativo. Esta población pertenece a familias con un mediano o bajo nivel económico y social.

Todos los estudiantes asisten a lecciones en compañía de un adulto, quien, por lo general, es su madre o, en algunos casos específicos, abuelas, hermanas o cuidadoras. La mayoría de estas madres se dedica a los quehaceres del hogar y sus edades se encuentran entre los 17 y 46 años.

\section{Cómo se hizo la investigación}

El estudio explora y describe la viabilidad de una intervención pedagógica basada en habilidades adaptativas, para los niños y las niñas del nivel de estimulación temprana, de acuerdo 
con las necesidades de los padres de familia y las del o la docente, con el fin de diseñar una propuesta más pertinente para los niños y las niñas de 0 a 3 años de edad, con discapacidad cognitiva del Centro de Enseñanza Especial de Heredia.

Se buscó conocer las necesidades y los intereses de los padres de familia en relación con el proceso educativo de sus hijos e hijas, por medio de un taller y la aplicación de un cuestionario.

Para determinar el nivel de conocimientos que tienen las docentes de estimulación temprana y de III y IV ciclos, sobre las habilidades y las conductas adaptativas, así como sobre la fundamentación y los principios que debe sustentar un plan de estudios desde esta perspectiva, se aplicó un cuestionario diseñado para cada uno de los niveles.

De acuerdo con la información recabada, se desarrolla una propuesta curricular para esta población en particular y se conforma la estructura de fases o etapas por seguir en la investigación, para dar una coherencia de Acción-Reflexión-Acción de la propuesta curricular, tal como se aprecia en la Figura 1.

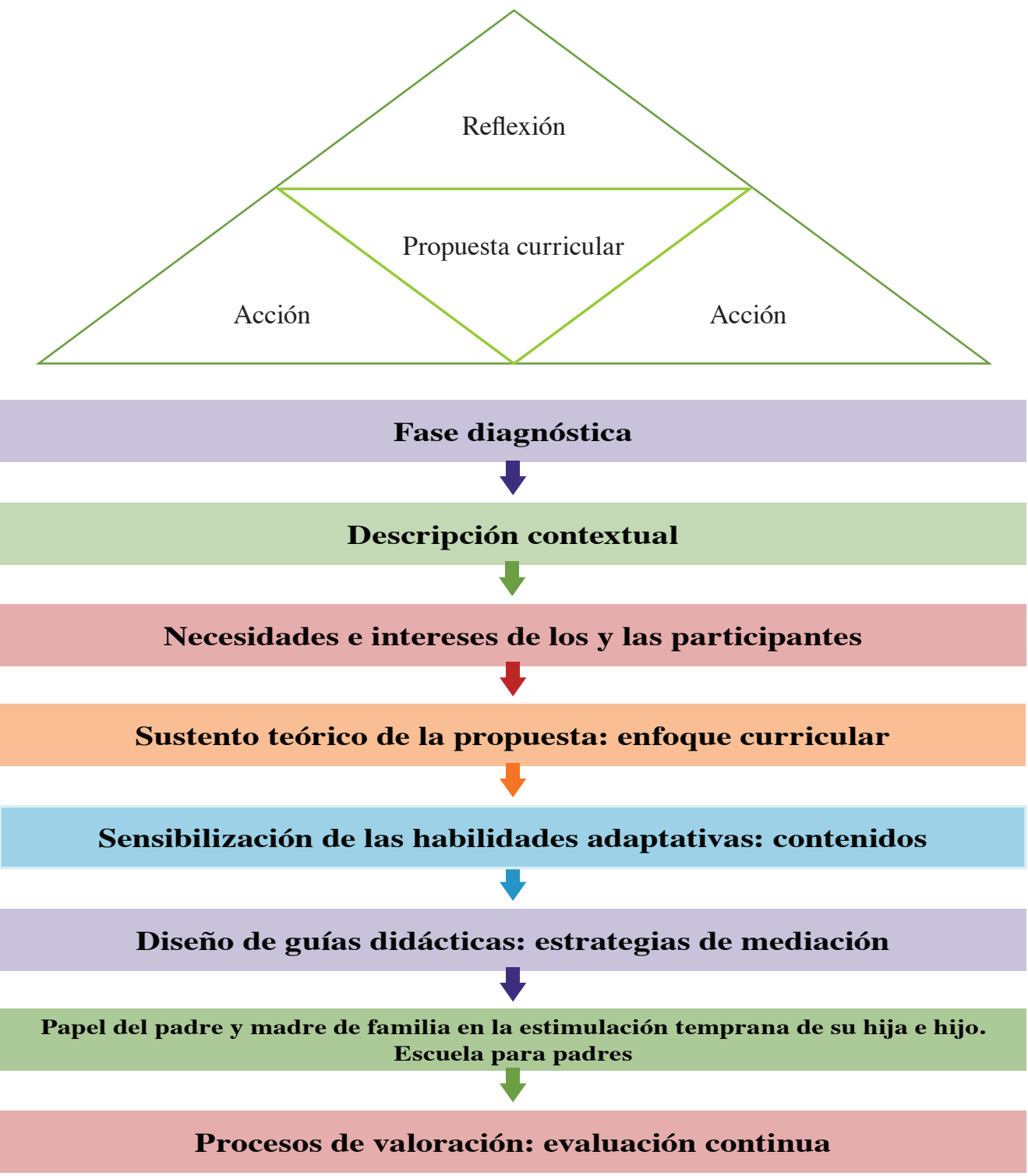

Figura 1. Estructuración de la propuesta curricular. Nota. Elaborada por Jara y Roda, en julio de 2008. 
La propuesta nació mediante la reflexión de la labor docente realizada con esta población durante muchos años, lo que generó una fase inicial de investigación del contexto social y educativo en que se desarrollaría (objeto de estudio); esto corresponde a la fase diagnóstica y la descripción contextual. La reflexión de esta acción lleva a determinar las necesidades e intereses de los y las participantes, y se realiza un proceso de acción-reflexión- acción para el desarrollo de cada una de las fases que sustentan la propuesta.

En la fase diagnóstica, se procedió a realizar un taller con los padres de familia, cuyo objetivo fue identificar sus intereses respecto a las acciones o conductas que desean que ejecuten sus hijos e hijas en esta etapa. Se les aplicó, además, un cuestionario que ayudó a sustentar la escogencia de las habilidades adaptativas prioritarias por abordar en este nivel (véase la Figura 2).

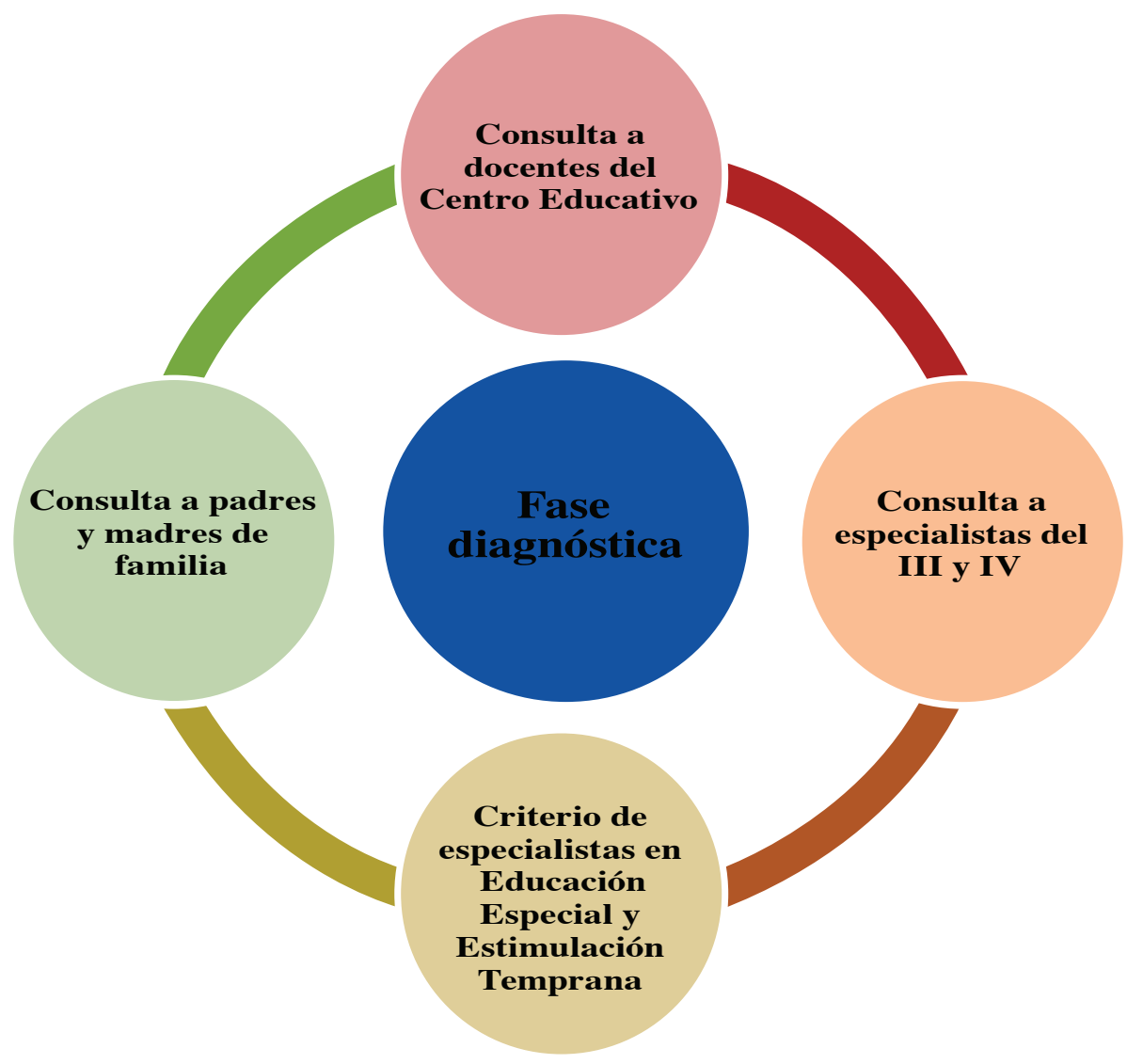

Figura 2. Organización de la fase diagnóstica.

Nota. Elaborada por Jara y Roda, en julio de 2008.

Como parte de la fase diagnóstica se aplicó un cuestionario a las docentes de estimulación temprana, con el fin de obtener su opinión con respecto a las conductas adaptativas que son prioritarias por desarrollar entre los 0 y los 3 años de edad. Esta técnica se utilizó, también, con las docentes de III y IV ciclos, para conocer su opinión respecto a las conductas que deben desarrollarse en los niños y las niñas que asisten a los servicios de estimulación temprana, para que puedan desempeñarse con mayor independencia en edades posteriores.

Además, se realizaron entrevistas a expertos (Zúñiga, 2008 y Esquivel, 2008) en el área de educación especial, para sustentar el desarrollo teórico de la propuesta. 


\section{Un breve sustento teórico}

Para entender el fundamento de la propuesta es necesario comprender el concepto de discapacidad, el cual es definido por Verdugo y Jenaro (2004), como "la expresión de las limitaciones en el funcionamiento individual dentro de un contexto, y representa una desventaja sustancial para el individuo" (p. 32). Es decir, la discapacidad se ve más marcada o disminuida, según la sociedad o el contexto en que se desenvuelva.

Al hablar de discapacidad cognitiva, Luckasson et al. (citados por Verdugo y Jenaro, 2004) la definen como: "una discapacidad caracterizada por limitaciones significativas en el funcionamiento intelectual y en la conducta adaptativa, que se manifiesta en habilidades adaptativas conceptuales, sociales y prácticas. Esta discapacidad comienza antes de los dieciocho años" (p. 17).

El funcionamiento intelectual debe entenderse en el contexto de las dificultades que experimentan las personas con retraso mental, para aprender y poner en práctica determinadas actividades de la vida diaria relacionadas con la inteligencia conceptual, práctica y social.

En un proceso de socialización, un individuo debe adaptarse a su entorno social y aprender a aceptar las demandas que éste imponga.

En el año 1992, la Asociación Americana de Retardo Mental (citada por Verdugo y Jenaro, 2004) asignó mayor relevancia a las habilidades de adaptación, especificó, por primera vez, diez posibles dimensiones de ella y propuso la siguiente definición:

Retraso mental se refiere a limitaciones substanciales del funcionamiento actual. Se caracteriza por un funcionamiento intelectual significativamente por debajo del promedio, que se presenta junto a limitaciones relacionadas con dos o más de las siguientes áreas de destrezas adaptativas: comunicación, cuidado de sí mismo, vida en el hogar, destrezas sociales, uso de recursos comunitarios, auto dirección, salud y seguridad, destrezas académicas funcionales, ocio y trabajo. El retraso mental se manifiesta antes de los 18 años. (p. 1)

Las personas que presentan mayor compromiso cognitivo serán, entonces, las que tengan los niveles más bajos de conducta adaptativa, por tanto, son las que requieren de mayor apoyo por parte de su entorno social y una mejor calidad educativa que les permita cubrir sus necesidades para potenciar sus posibilidades. Verdugo y Jenaro (2004) dividen los apoyos en:

Intermitente: Apoyos "cuando sea necesario", caracterizados por su naturaleza episódica ... pueden ser de alta o baja intensidad.

Limitado: Intensidad de apoyos caracterizada por su consistencia a lo largo del tiempo, se ofrecen por un tiempo limitado ..., pueden requerir menos personal y costos que niveles de intensidad de apoyos más intensos ...

Extenso: Apoyos caracterizados por la implicación regular ... en al menos algunos ambientes ...

Generalizados: Apoyos caracterizados por su constancia, elevada intensidad, provisión en diferentes ambientes, puede durar toda la vida. (pp. 187-189) 


\section{Habilidades adaptativas desde una perspectiva teórica de Vigotsky}

El aprendizaje y el desarrollo interactúan desde los primeros días de la vida del niño y de la niña; el aprendizaje guía al desarrollo estimulando áreas cerebrales y estableciendo conexiones neuronales que enriquecen el pensamiento lógico, el lenguaje, la memoria y la atención.

En el desarrollo del niño y de la niña, debe tenerse en cuenta su mundo social, el cual influye en las habilidades lingüísticas y cognitivas; Becco (2000) opina que:

Vigotsky señala que el desarrollo intelectual del individuo no puede entenderse como independiente del medio social en el que está inmersa la persona. Para Vigotsky, el desarrollo de las funciones psicológicas superiores se da primero en el plano social y después el nivel individual. La transmisión y adquisición de conocimientos y patrones culturales es posible cuando de la interacción -plano interpsicológico- se llega a la internalización -plano intrapsicológico. (p. 1)

El aprendizaje se produce más fácilmente en situaciones colectivas, en las que los padres del niño o de la niña son los primeros en favorecer el aprendizaje. Además, las influencias ambientales dan paso a la formación de estructuras mentales más complejas.

Para Vigotsky, la discapacidad no debe limitar el aprendizaje, sino que estos niños y estas niñas tienen capacidades que pueden desarrollar en forma diferente; la discapacidad de una persona resulta de la interacción entre la persona y el ambiente en el que vive, y en el cual la familia constituye el primer ambiente donde se desarrolla.

La sociedad tiene códigos culturales propios que, a veces, a las personas con retardo mental se les imposibilita decodificar para poder adaptarse a la realidad. Por consiguiente, el mejor progreso y adaptación a su entorno social dependerá de la ayuda que brinde ese mismo medio social en que se desarrolla.

La socialización se realiza en forma progresiva desde que el niño o la niña nace, y la primera interacción la hace con la madre, luego con el padre y el resto de la familia.

Desde la visión de Vigotsky, la mejor enseñanza es la que se adelanta al desarrollo, la que le da mucha importancia a la interacción del niño o la niña; en ella, los mediadores del aprendizaje (familiares, educadores u otros) influyen para ayudarle a desarrollar sus capacidades cognitivas, las cuales, según nuestra experiencia, requieren de una serie de habilidades adaptativas en el caso de los y las estudiantes con discapacidad cognitiva. De esta forma, se habla de la Zona de Desarrollo Próximo que evidencia lo que el estudiante puede lograr por sí mismo, y lo que puede llegar a hacer con la ayuda de un adulto. Vigotsky (citado por Tryphon y Vonèche, 2000) la define como "la diferencia entre el nivel evolutivo real [del niño] según lo determina una resolución independiente de problemas [y su] desarrollo potencial determinado mediante la resolución de problemas con guía adulta o en colaboración con pares más capaces" (p. 227). El potencial se define como el nivel de competencia que un infante puede alcanzar, cuando es guiado y apoyado por otra persona.

Se destaca siempre la idea de que es mejor que al niño y a la niña los guíe un adulto o persona significativa en su vida, quien será mediadora entre la destreza que va a adquirir, entiéndase esto como el andamiaje, que como lo mencionan Papalia, Wendkos y Duskin (2005, p. 37) "es el apoyo temporal que los padres, maestros u otros dan al niño para realizar una tarea, hasta que el niño puede hacerlo por sí mismo".

Desde el concepto de la discapacidad, se entrelazan las teorías de Vigotsky, por ser compatibles con las propuestas educativas basadas en las habilidades adaptativas, que se deben ejecutar en la práctica desde el nacimiento del niño o de la niña, en condición de discapacidad. 
Vigotsky hace énfasis en la importancia de la educación de los niños y las niñas desde la cuna y en su medio social, lo cual, también, se recalca en la educación especial. Expresado de otra forma: las habilidades adaptativas y su implementación en el hogar, desde la más tierna infancia, son congruentes con las teorías educativas propuestas por él.

Las habilidades que desarrolla el infante se convierten en el conocimiento previo y la conducta adaptativa es el conocimiento deseado. Al respecto, Zuñiga (2008) comenta que "la habilidad es la capacidad que va desarrollando el individuo para lograr ciertas cosas ... lograr hacerlas, esa es la conducta". Éste es el fin de la educación especial, crear independencia por medio del desarrollo de nuevas habilidades, de acuerdo con sus capacidades.

\section{Familia del estudiante con discapacidad cognitiva: integración del contexto inmediato del estudiante}

Es importante que la familia se involucre en el proceso educativo de los bebés desde el nacimiento y, para ello, debe tener muy claro cuáles son los objetivos y las acciones que deben realizar, tal como lo describe Peralta (2002) cuando menciona que "la concepción de la educación parvularia como un saber que apoya y complementa profesionalmente la educación familiar, es insustituible por sus diferentes aportes” (p. 140).

La importancia del establecimiento de un vínculo amoroso fuerte y confortable con el niño o la niña, una figura de apego de cara a un desarrollo óptimo de la persona ha sido subrayada por psicodinámicos (rama del psicoanálisis), quienes opinan que el desarrollo emocional se construye en la estructura del cerebro de los niños como respuesta a su experiencia personal y por las influencias de los ambientes en los que viven; de manera que durante el proceso de desarrollo infantil, sus experiencias emocionales tempranas están, literalmente, caladas en la arquitectura de sus cerebros.

Este desarrollo del apego durante la primera infancia, marca la importancia de trabajar los programas de estimulación temprana con el padre y la madre dentro del aula, ya que no resulta conveniente para el desarrollo socio-emocional del párvulo, romper con dicho vínculo.

Al fortalecer el vínculo afectivo y el apego madre-hijo y padre-hijo, también se están viendo favorecidas las demás áreas del desarrollo; ya que si el ser humano es un ser integral, al sufrir una alteración en el área socio emocional, las demás áreas del desarrollo también se verán afectadas.

La familia tiene una gran relevancia como agente educativo, por lo que el profesional en educación debe guiar a los padres, las madres y encargados, sobre cómo enseñar a sus hijos y potenciar, con ello, sus capacidades. Los padres son las personas que siempre alientan, enseñan y proporcionan elogios y refuerzos positivos.

La familia, en general, tiene la tarea de enseñar cientos de capacidades, nadie conoce a un infante mejor que sus padres, ni tiene tanto interés en éste como ellos.

\section{Un currículo asertivo para los y las estudiantes en condición de discapacidad cognitiva}

Existen diferentes concepciones de currículo, las cuales, en su mayoría, coinciden en que es el resultado de todas las experiencias que los educandos acumulan para su desarrollo a nivel integral, como lo dice Zúñiga “... tanto desde el punto de vista biológico, psicológico, social, político y económico” (2008).

Por tanto, el currículo es un medio de aprendizaje, orientado por diferentes posiciones filosóficas, políticas, ideológicas y paradigmáticas, que busca la concretización de la política educativa según los fines y los objetivos de la educación. 
Este proceso se lleva bajo la planificación del docente, con el propósito de que el estudiante se desempeñe socialmente, lo cual, en Educación Especial se puede entender como se muestra en la Figura 3.

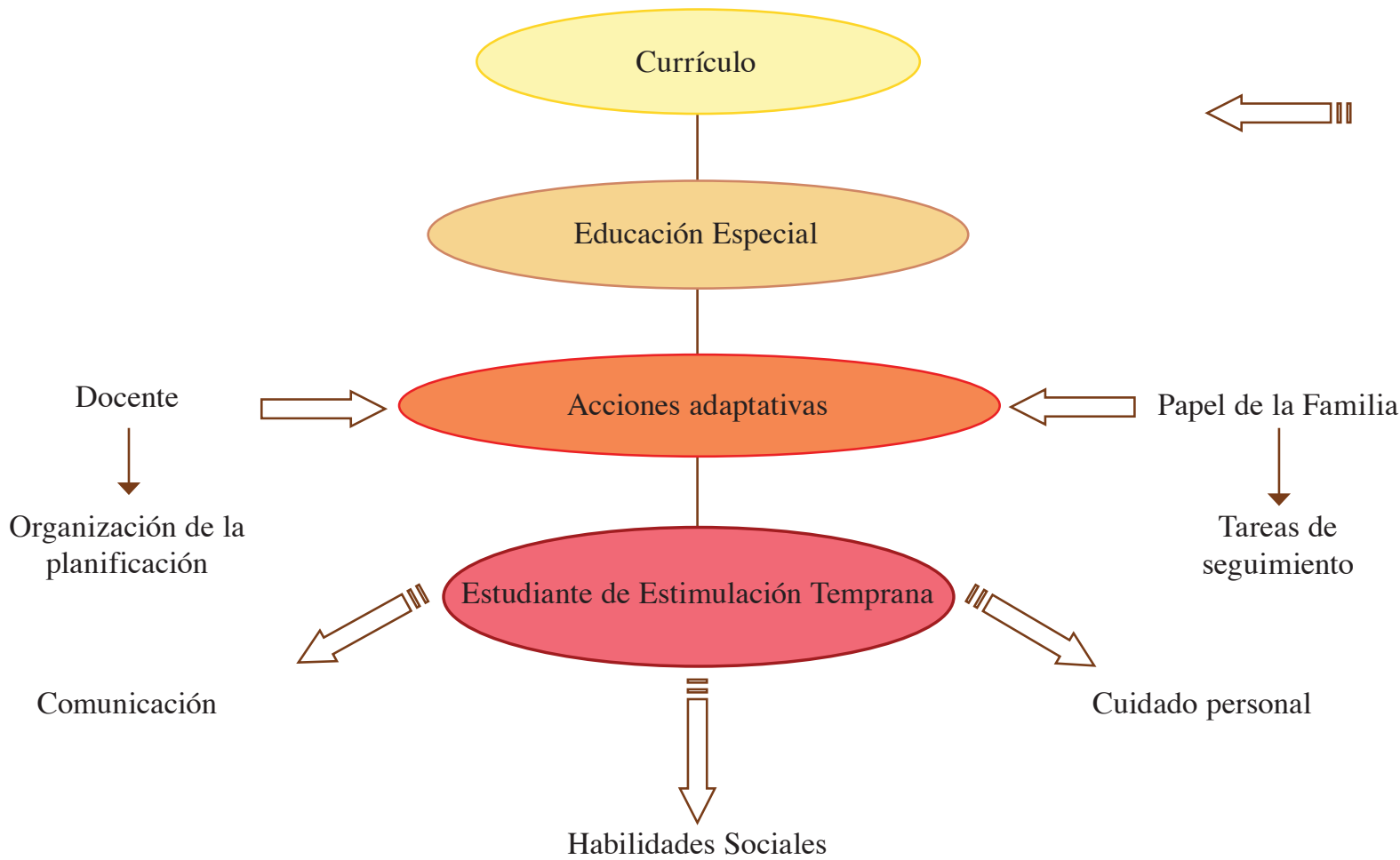

Figura 3 Estructura curricular en Educación Especial.

Nota: Elaborada por Jara y Roda en Julio, 2008.

Con la propuesta se busca que el currículo de educación especial esté basado en acciones adaptativas que involucren al docente y al padre de familia de una forma lineal. El docente, desde la organización, la planificación y la puesta en práctica de los objetivos, y el padre de familia en la ejecución en el hogar de las tareas que favorezcan la adquisición, por parte del estudiante, de las habilidades adaptativas; las cuales, en el caso del nivel de estimulación temprana, serían la comunicación, las habilidades sociales y el cuidado personal.

\section{Las habilidades adaptativas incluidas en la propuesta curricular}

Durante el proceso de estimulación temprana con los niños y las niñas de 0 a 3 años de edad, se considera primordial el desarrollo de las habilidades adaptativas más básicas en esta etapa, como son las de comunicación, las habilidades sociales y el cuidado personal o de sí mismo, ya que una sustenta, evolutivamente, las otras, es decir, no existe comunicación sin socialización y viceversa: la socialización sin comunicarnos; en ellas, el contexto influye directamente en lo que hacemos y somos como individuos.

La comunicación es indispensable en todas las etapas de la vida del niño o la niña; un problema en esta área afecta la interacción con las personas con las que se relaciona y perturba o interrumpe el proceso de socialización natural (proceso natural en que el ser humano va adquiriendo las normas y 
los valores de convivencia que estructuran su personalidad, manera de pensar, conductas e identidad, para vivir en sociedad).

Las dificultades para comunicarse afectan el proceso de socialización natural, el cual se lleva a cabo, en primera instancia, dentro de la familia, el entorno escolar y el ambiente social.

Sobre esto, Zúñiga (2008) opina que “... la expresión de sentimientos, emociones, opiniones, ideas, pensamientos, etc., está fuertemente asociada a la experimentación de autonomía, autorrespeto y autovaloración".

Cuando se habla de habilidades sociales en etapas tan tempranas, se hace referencia a las interacciones que establece el párvulo con su padre, su madre, sus hermanos u otros miembros de la familia, asimismo, con otras personas, tanto niños o adultos de su comunidad y entorno escolar.

El desarrollo de estas habilidades de comunicación y socialización van a requerir, por parte del menor, cierto grado de independencia, por lo cual resulta sumamente importante desarrollar en el niño o la niña destrezas de autocuidado, de acuerdo con su etapa de desarrollo, que le permitan adaptarse a su entorno social.

Por ello, dentro de la estimulación temprana son contemplados los diversos contextos en que se ve inmerso el niño o la niña, para, así, desarrollar las habilidades adaptativas oportunas, precisas y, ante todo, competentes a las exigencias de las demandas sociales futuras e inmediatas, para formar un talento humano.

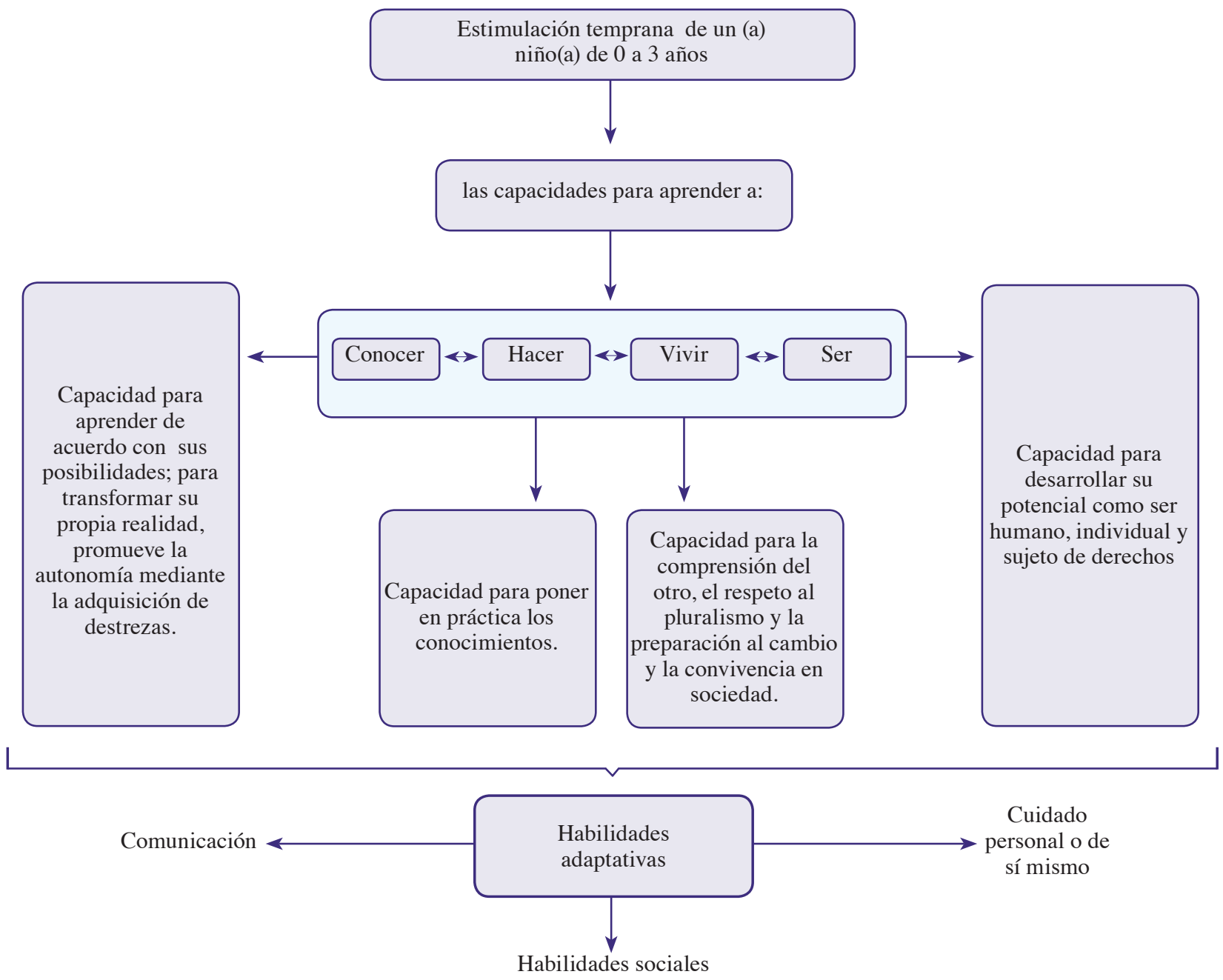

Figura 4. Talento humano de Educación Especial de 0 a 3 años

Nota. Tomado de Delgado (2007) y adaptado por las autoras. 
La estimulación temprana tiene como fin primordial desarrollar las capacidades naturales de los niños y las niñas: conocer, hacer, vivir y ser; tal como se describen en la Figura 4.

Estas capacidades son esenciales para la adquisición y el desarrollo de las habilidades adaptativas mencionadas anteriormente; y son básicas en estas edades.

\section{Diseño de la propuesta curricular: inventario ecológico de nuestras metas y fines}

El diseño de la propuesta curricular está dirigido a los procesos de adquisición de las habilidades adaptativas, en este caso de los niños y las niñas en condición de discapacidad cognitiva de 0 a 3 años de edad. Para ello, se propone conformar un ciclo de acción-reflexión-acción con participación de docentes, estudiantes y padres de familia.

\section{Propósitos de la propuesta}

Esta propuesta curricular busca llevar al niño y la niña, en condición de una discapacidad cognitiva, al máximo desarrollo de las potencialidades cognitivas, sociales, emocionales y físicas.

\section{Objetivo General}

Propiciar el desarrollo integral del niño y la niña con discapacidad cognitiva de cero a tres años de edad, a fin de que logre potencializar sus habilidades adaptativas: sociales, de comunicación y de cuidado personal o de sí mismo; dentro del contexto educativo y familiar.

\section{Objetivos específicos}

Habilidades sociales

1. Favorecer el proceso de adaptación, socialización y valores de cada niño y niña, en su entorno.

2. Fortalecer la inteligencia emocional y múltiple con el desarrollo de las habilidades que conformen los rasgos del carácter del niño y la niña.

Comunicación

1. Estimular el desarrollo del lenguaje, las distintas formas de comunicación y representación del niño y la niña.

2. Enriquecer el vocabulario y la capacidad de expresión en los niños y las niñas.

Cuidado personal o de sí mismo

1. Fortalecer el desarrollo de los hábitos de alimentación e higiene en los niños y niñas con discapacidad cognitiva. 


\section{Sensibilización de las habilidades adaptativas en la propuesta: contenidos}

Esta propuesta curricular está conformada por tres partes:

1. Dirigida a la docente del nivel de estimulación temprana de niños y niñas de cero a tres años de edad, con discapacidad cognitiva. Consiste en un plan de estudio con objetivos generales y específicos, así como los contenidos para cada una de estas habilidades, divididos en tres etapas. El o la profesional que lo aplique tendrá la libertad de diseñar las estrategias de mediación de acuerdo con las necesidades y las características de la población estudiantil que atiende, así como los recursos con los que cuenta.

2. Dirigida a los padres, las madres y demás miembros del grupo familiar. Consiste en un fichero de tareas para ser ejecutadas por la familia en el hogar, las cuales contienen una serie de actividades, divididas por clave color, según la habilidad a la que respondan. La familia deberá realizar en el hogar estas tareas, las cuales serán adecuadas a las necesidades e intereses del contexto familiar y del niño o la niña.

3. Consta de una serie de instrumentos de evaluación y de control de avance para la docente y la familia; contiene una lista de cotejo o perfil con las acciones que el niño o la niña irá adquiriendo durante el proceso educativo, con los cuales la familia podrá ir evidenciando el avance del menor con base en los rubros de logrado (L) y logrado con ayuda (LA). Además, la propuesta incluye un instrumento de evaluación de avance bimensual para el hogar, el cual será la base de trabajo para la Escuela para padres, que se llevará a cabo al final de cada bimestre.

El objetivo de la Escuela para Padres es brindar a los miembros de la familia un espacio para compartir sus experiencias en la ejecución de las tareas con otros padres de familia, y crear, así, una línea de realimentación, entre padres y docente.

Con base en los comentarios o dudas que planteen o expongan los y las participantes, se podrán realizar los ajustes y las modificaciones necesarias, de manera que los objetivos puedan desarrollarse en cada caso particular, según las necesidades de cada estudiante.

Es importante que se comprenda la lógica del desarrollo de los contenidos por las habilidades adaptativas estructuradas por etapas. Estas tres etapas siguen la línea de desarrollo integral del menor.

La primera es la Etapa Bebé, que comprende desde el nacimiento hasta, aproximadamente, un año de edad. La segunda, que llamaremos Etapa Infante abarca el segundo año de vida y, por último, la Etapa Maternal, la cual abarca el tercer año de vida.

Cada una de estas etapas aborda los contenidos y los objetivos de las habilidades adaptativas que irá adquiriendo el niño o la niña mediante una secuencia lógica de acuerdo con su crecimiento y su desarrollo.

\section{Diseño de las guías didácticas: estrategias de mediación}

La mediación pedagógica (entendida como: aprender a conocer, aprender a hacer, aprender a vivir juntos y aprender a ser) fundamenta esta propuesta curricular.

Esta mediación pedagógica utiliza estrategias como la relación del tema con la experiencia particular del participante (niños, niñas, padres de familia y docentes) y de otros en un contexto social determinado; la 
estimulación de preguntas como un proceso que conduce a resultados, conclusiones y compromisos para la práctica; en la que la educación se enfoca, entonces, desde la perspectiva de potenciar el desarrollo del niño o la niña, ayudando a desarrollar las habilidades adaptativas de comunicación, habilidades sociales y cuidado personal o de sí mismo, para que quien aprende, sea capaz de construir y reconstruir su aprendizaje.

Todo ello se logra por medio de una interacción horizontal entre docente y padres, quienes deben abordar las diversas temáticas relativas al desarrollo integral del niño y la niña, de manera que la familia logre empoderarse de los procesos de enseñanza y de aprendizaje de su hijo o hija; tal como puede observarse en la Figura 5.

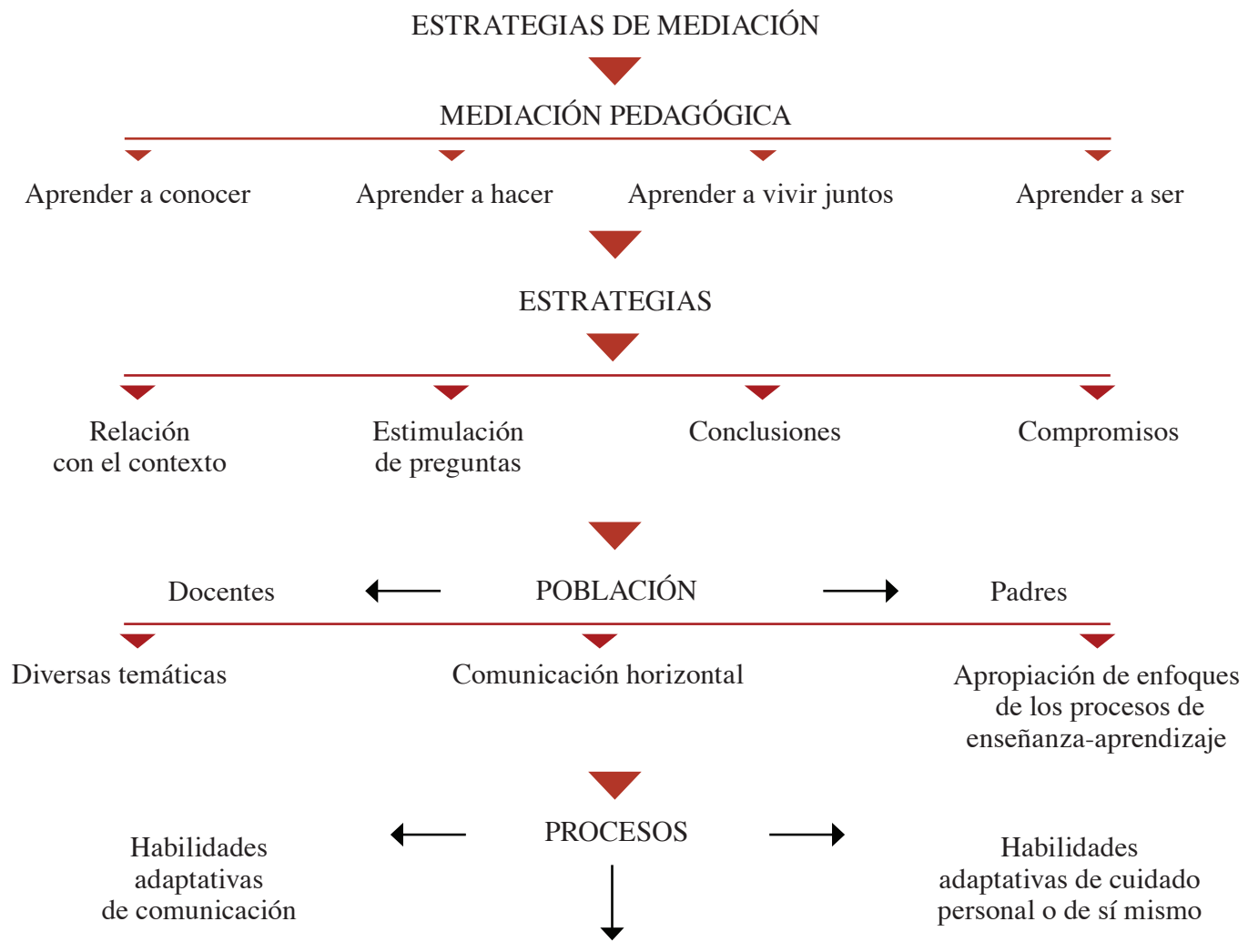

Habilidades

adaptativas

de socialización

Figura 5: Nuevos Enfoques de los procesos de enseñanza- aprendizaje.

Nota. Tomado de Delgado (2007) y adaptado por las autoras.

La metodología del diseño curricular basado en habilidades adaptativas, fomenta el potencial crítico y creador de las personas involucradas, ubica al profesional como elemento de apoyo al promover la independencia y la democratización del pensamiento, y abre espacios para la toma de decisiones, para fomentar procesos cuyos componentes son decididos por los propios participantes. Estos componentes responden a las sugerencias expresadas en la investigación, las cuales se fundamentan en las necesidades de validar las habilidades adaptativas propuestas.

Para implementar y validar la propuesta se diseñó una guía para los y las docentes de estimulación temprana de niños y niñas con discapacidad cognitiva, partiendo de las habilidades adaptativas seleccionadas en este estudio, considerando los hallazgos y los criterios desde la fase diagnóstica citados por los y las participantes, así como las tareas para padres y madres de familia. 


\section{Procesos de valoración: evaluación permanente de la propuesta curricular}

La evaluación consiste en comparar lo logrado mediante los objetivos planteados. En esta propuesta, la evaluación se retoma en la Acción-Reflexión-Acción por parte de la docente respecto a las habilidades adaptativas alcanzadas en el proceso de planificación.

En el proceso de evaluación, basado en habilidades adaptativas, el programa, continuamente, debe estar considerando el aprendizaje, evidenciando y fortaleciendo el dominio del conocimiento en la comunicación, socialización y cuidado personal o de sí mismo y el resultado del aprendizaje del educando, considerando la observación y el análisis del proceso de trabajo desempeñado por los niños y las niñas, lo que demuestra una adquisición práctica de lo integrado al desempeño mismo (situaciones reales).

La proyección de la evaluación basada en habilidades adaptativas es el realimentar a los padres de familia, con respecto a la estimulación de las destrezas de sus hijos e hijas de cero a tres años de edad, que asisten al nivel de estimulación temprana, hacia la satisfacción de sus necesidades y expectativas. Para esto, dentro de la propuesta se han elaborado dos instrumentos, por lo que la propuesta curricular, en cuanto a la evaluación basada en habilidades adaptativas, establece:

1. Procesos de selección de habilidades adaptativas considerando los criterios (cuestionarios y entrevistas)

2. Asesoramiento y capacitación a los profesores en el enfoque por habilidades adaptativas

3. Diseño de criterios para valorar los procesos de adquisición de las habilidades que se observan

4. Ayuda a la implementación y al cumplimiento, realimentando constantemente la propuesta, considerando las experiencias académicas vivenciadas por el profesorado facilitador y facilitadora, y el padre de familia participante

5. Vela por la reflexión de lo hecho y lo esperado

\section{HABILIDADES ADAPTATIVAS}

Capacidad para cumplir una tarea o actividad conllevando una

\section{Acción- Reflexión- Acción}

\section{Comunicación}

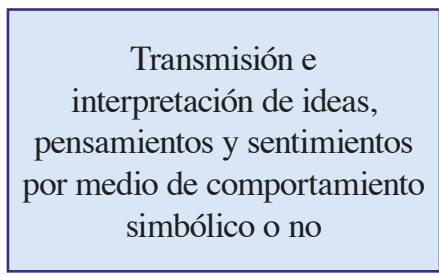

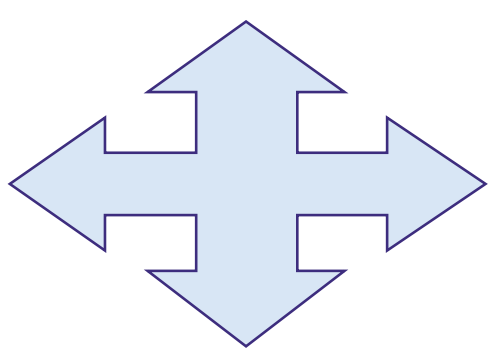

Habilidades sociales

Destrezas para establecer y mantener relaciones afectivas con los demás
Cuidado personal o de si mismo

Acciones destinadas al mejoramiento y bienestar del ser humano, en cuanto a salud, higiene y alimentación

Figura 6: Propuesta curricular a partir de las habilidades adaptativas Nota: Elaborada por Jara y Roda en Julio, 2008. 
Un proceso evaluativo desde las habilidades adaptativas valora la capacidad del estudiante para ejecutar ciertas destrezas adaptativas, relacionadas o inmersas en alguna de las tres habilidades detalladas en la Figura 6. Con base en la organización (saber actuar), la planificación (saber conocer) y la ejecución (saber hacer), se llega a la evaluación (saber pensar). El profesional en educación especial combina la teoría y la práctica dentro del contexto de los estudiantes para la ejecución de la propuesta.

\section{Puesta en práctica: cronograma}

La planificación educativa del nivel de estimulación temprana para niños y niñas de 0 a 3 años de edad, con discapacidad cognitiva, que asisten a un centro de enseñanza especial, se lleva a cabo a partir de un diagnóstico que realiza el o la docente al inicio del curso lectivo.

Para la ejecución del proceso diagnóstico, se establece un período de cuatro a seis semanas, tomando en cuenta que los y las estudiantes de este nivel asisten a clases una, dos o tres veces por semana.

La o el docente contará con un perfil de funcionamiento de acuerdo con la etapa de desarrollo en la que se encuentre el o la estudiante, el cual será implementado en el aula y complementado con la visita al hogar, donde se evalúa al estudiante dentro de su contexto real, para obtener la información necesaria con el fin de diseñar el plan individual semestral del estudiante.

Esquivel (2008) menciona que en su práctica educativa “... se trabaja en conjunto permitiéndole al padre, al inicio del curso, proponer objetivos que consideren importantes de ser abordados con sus hijos ... y formarán parte del plan individual del o la estudiante".

Por ello, en la visita al hogar se realiza una entrevista a la madre, al padre o al encargado del o de la menor, esto con el objeto de conocer la situación socioeconómica de la familia e indagar en las necesidades e intereses que tiene ésta con respecto al desarrollo y crecimiento del niño o la niña.

Una vez recabada la información y terminado el diagnóstico individual, la o el docente contará con la información suficiente para elaborar el planeamiento individual semestral de cada estudiante, el cual debe tener contenidos desglosados en objetivos específicos para cada una de las habilidades adaptativas: comunicación, habilidades sociales y cuidado personal o de sí mismo. Este documento se adjunta al expediente acumulativo del o la estudiante, y debe estar firmado por el padre de familia o encargado.

Como documento de control de avance del proceso, y guía de las habilidades adaptativas adquiridas por el niño o la niña durante el proceso educativo, se cuenta con un perfil o lista de cotejo de las acciones, distribuidas en las tres habilidades adaptativas objeto de estudio, que el menor irá adquiriendo durante los años que permanezca en este nivel.

El perfil mencionado se llena con base en los criterios de logrado y logrado con ayuda, entendiendo este último como aquel objetivo que se encuentra en proceso de lograrse de forma independiente.

También con este perfil, la o el docente confecciona su planeamiento individual y grupal, para cada uno de los subgrupos que atiende, de acuerdo con las necesidades e intereses que haya detectado en sus estudiantes y sus familias, como parte de los principios de las diferencias individuales dentro del contexto educativo especial.

El o la docente deberá realizar un planeamiento semanal para cada subgrupo, basado en una programación previa. Además, debe elaborar una crónica semanal para cada subgrupo que atiende en la que destaque las fortalezas e inconvenientes que se le hayan presentado con su planificación y su ejecución, lo que le permitirá llevar a cabo un proceso de reflexión-acción-reflexión de su labor, para poder mejorar la intervención pedagógica inmediata. 
El o la docente ofrece a los padres de familia un perfil personalizado de su hijo o hija, según el plan individual. Asimismo, se les da una serie de tareas bimensuales que deben aplicar en el hogar para apoyar el plan individual.

Cada dos meses, se debe efectuar una Escuela para padres, en la que los y las participantes llevan su carpeta de control e inquietudes; de esta forma, se hace la realimentación entre todos y todas, con el fin de compartir experiencias, aciertos y obstáculos que se hayan encontrado durante el proceso de trabajo con sus hijos o hijas.

Con los aportes dados por los y las participantes, el o la docente podrá asignar las nuevas tareas para los siguientes dos meses.

Se considera que durante el año se llevarán a cabo de cuatro a cinco Escuela para padres, como parte de la necesidad de ampliar la información del progenitor en el cuidado y la estimulación de su hijo o hija.

En el mes de julio, la o el docente debe entregar al padre de familia un informe de avance de medio período, el cual consiste en una lista de cotejo de los objetivos abordados. Este documento cuenta con un apartado de observaciones y recomendaciones generales a la familia. El diseño de dicho documento queda sujeto al criterio y gusto de la docente, y debe adjuntarse una copia firmada por el padre de familia o encargado al expediente del estudiante. Con base en los resultados de esta evaluación de medio año, el o la docente confeccionará el plan individual del segundo semestre.

Al finalizar el curso lectivo, en el mes de diciembre, la docente realizará un informe final, el cual debe redactarse de manera descriptiva, resaltando las habilidades adquiridas por el niño o la niña hasta ese momento. Como lo establece el MEP (2005), este informe “... tiene como finalidad comunicarle a los padres o encargados los logros de sus hijos, en los períodos establecidos por el Calendario Escolar" (p. 83).

De la misma forma, contará con un apartado para observaciones y recomendaciones. Una copia de este documento firmada por el padre de familia o encargado se anexará al expediente acumulativo del estudiante.

\section{Consideraciones finales}

Esta propuesta ofrece la posibilidad, a los centros de educación especial, de diseñar una respuesta educativa acorde con las características y necesidades particulares del estudiante, de trabajar por la igualdad de oportunidades y de ofrecer a los y las estudiantes el fortalecimiento de sus habilidades para el desarrollo de sus potencialidades. Igualmente, el brindar la posibilidad de conseguir una educación integral, mejorando las destrezas y las habilidades adaptativas, así como los valores éticos, morales y ciudadanos que deben tomarse en cuenta en una educación de calidad, desde la perspectiva ecológica en el campo de la educación especial.

Con base en la propuesta anterior, los y las docentes que trabajen en estimulación temprana, con una población con discapacidad cognitiva, podrán contar con un documento que les permitirá desarrollar mejor su labor educativa.

La propuesta puede ser implementada tanto por las universidades (públicas o privadas), en el proceso de formación de los y las docentes, como por las actuales profesionales en educación especial como una estrategia de innovación en su labor docente.

Se debe recalcar que la propuesta toma en cuenta la participación de la familia en los procesos de enseñanza y de aprendizaje, al igual que el contexto real del estudiante, con el fin de mejorar la calidad de la educación que se brinda. 
Vivimos una realidad en la que presentar una discapacidad, continúa siendo motivo de exclusión, ya que se cree que estas personas no poseen las habilidades para pertenecer a una sociedad de consumo, materialismo, individualismo y egocentrismo. Por eso, resulta de gran relevancia el lograr que nuestros estudiantes adquieran las destrezas necesarias para ser personas independientes, según sus necesidades especiales, y que logren insertarse, ser aceptados y respetados dentro de su contexto familiar y social.

Para ello, debemos iniciar un trabajo que fomente el desarrollo integral de los niños y las niñas desde las edades iniciales, para que puedan, conforme vayan creciendo, superar las dificultades que se les presentan. La estimulación temprana dentro de la educación especial juega un papel de esperanza para las familias de niños y niñas que presentan una discapacidad cognitiva como una forma de mejorar su calidad de vida.

La propuesta aporta una herramienta teórica y práctica para los y las docentes de estimulación temprana y brinda la oportunidad de involucrar a la familia durante todo el proceso.

\section{Referencias bibliográficas}

Becco, G. (2000). Vigotsky y las teorías del aprendizaje. Recuperado el 25 de setiembre, 2008, de http://perso.wanadoo.es/angel.saez/pagina_nueva_165.htm

Costa Rica. Ministerio de Educación Pública. Departamento de Educación Especial. (2005). Normas y procedimientos para el manejo técnico-administrativo de los servicios educativos para estudiantes con retardo mental. San José, Costa Rica: Litografía e Imprenta LIL.

Delgado, W. (2007). Propuesta curricular para la Maestría Profesional en ciencias de la educación con énfasis en administración educativa basada en competencias Trabajo final de investigación aplicada del programa de estudios de posgrado en educación. San José, Costa Rica: Sede Rodrigo Facio de la Universidad de Costa Rica.

Esquivel, M. (2008, Setiembre). Currículum para la Educación Especial. Entrevista. Heredia, Costa Rica: Universidad Nacional.

Papalia, D., Wendkos, S. \& Duskin, R. (2005). Psicología del desarrollo. De la infancia a la adolescencia. ( $9^{\mathrm{a}}$ ed.). México, D. F.: McGraw Hill.

Peralta, M. (2002). Una pedagogía de las oportunidades. Nuevas ventanas para los párvulos latinoamericanos del siglo XXI. Santiago, Chile: Editorial Andrés Bello.

Tryphon, A. \& Vonèche, J. (2000). Piaget-Vigotsky: la génesis social del pensamiento. Buenos Aires, Argentina: Paidós.

Verdugo, N. \& Jenaro, C. (2004). Retraso Mental. Definición, clasificación y sistemas de apoyo. (10 ed.). Madrid, España: Alianza Editorial.

Zúñiga, E. (2008, Octubre). Currículum para la Educación Especial. Entrevista. Heredia, Costa Rica: Universidad Nacional. 